\title{
Time Reversal Direction of Arrival Estimation With Cramér-Rao Bound Analysis
}

\author{
Foroohar Foroozan and Amir Asif \\ Computer Science and Engineering \\ York University, Toronto, Canada M3J 1P3 \\ Email: \{foroozan, asif\}@cse.yorku.ca
}

\begin{abstract}
In this paper, the effect of coupling time reversal (TR) to direction of arrival (DOA) estimation is studied through theoretical Cramér-Rao bound (CRB) analysis and numerical simulations. The proposed TR/DOA estimator adds an additional stage and retransmits the time-reversed versions of the observations made during the original forward probing stage. The backscatters of the time reversed probing signals obtained from this second TR stage is used for DOA estimation based on the Capon algorithm. Simulations results and CRBs comparing the performance of the proposed TR/DOA estimator with that of the conventional approach based only on observations from the forward probing stage are presented.
\end{abstract}

Index Terms-Time Reversal, Cramér-Rao Bounds, DOA Estimation, and Multipath.

\section{INTRODUCTION}

Direction-of-Arrival (DOA) estimation is an important research topic in array signal processing with many applications in radar, sonar, and communications. In radar and sonar systems, the DOA information is used to localize the position of a target. In cellular and communication networks [1], DOA estimation aids in improving the performance of the network by eliminating cochannel interferences and is, therefore, proposed as one of the main function requirements for directionfinding smart antennas [2] in the next generation mobile communication systems. An array works on the premise that the desired signal and unwanted cochannel interferences arrive from different directions with most cellular systems using two separate frequencies for the transmit and receive modes. The knowledge of the DOA from the receive mode may be used during the transmit mode to adjust the beam pattern of the array and focus the signal strength in the direction of the communicating cellular mobiles. By directing the nulls toward mobiles in the other cochannel cells, interference within these cochannel cells can be significantly reduced. This leads to greater spatial diversity in multi-user environments.

The paper proposes a new scheme for DOA estimation based on time reversal (TR) [3], [4]. In conventional DOA range estimators based on the direct-path-only propagation observations, multipath is often ignored or considered detrimental to the performance of the estimators. In this paper, we take a different approach and address the question of what can be gained if a DOA estimator explores the additional information existing in the temporal structure of the observed field (i.e., in the set of multipath delays) to its advantage. Our approach is based on a TR setup and does not assume any particular multipath model. During the forward probing step, an active array of antennas, $(1 \leq k \leq P)$, receives the superposition of several attenuated and delayed replicas of the backscattered fields from a passive target. This observation vector $\boldsymbol{r}_{k}(t)$ recorded at the antenna array during the forward probing step is used in the conventional DOA estimators. Our TR/DOA estimator adds an additional probing stage by time reversing $\boldsymbol{r}_{k}(t)$ and retransmitting the time reversed signals to probe the channel a second time. The backscatters of the time reversed probing signals (obtained from the second TR stage) is used for DOA estimation in the proposed TR/DOA estimation algorithm.

To compare the performance of TR/DOA estimator with the conventional approach, we apply the wideband Capon [5]-[7] for estimating the location of the target. In other words, the wideband observations are decomposed into many narrowband bins using filter banks or the discrete Fourier transform before applying the narrowband Capon to each bin. The objective of the paper is not so much to devise a DOA estimation procedure based on Capon but to quantify the improvement, if any, with the application of TR to DOA estimators. The second contribution of the paper is to derive analytical expressions for the Cramér-Rao bounds (CRB) for the conventional and TR/DOA estimators. The CRBs illustrate the potential of improved performance with TR/DOA estimators, while Monte Carlo simulations are used to compare the actual performances. In our simulations, the TR/DOA Capon estimator outperforms conventional Capon by a wide margin especially at low signalto-noise ratios.

The organization of the paper is as follows. Section II defines the notation and derives the observation models for the conventional and TR/DOA estimators operating in a rich multipath environment. The Capon implementation of the TR estimator is discussed in Section III, while analytical expressions for the CRBs for the conventional and TR Capon algorithms are derived in Section IV. Results from Monte Carlo simulations are discussed in Section V. Finally, Section VI concludes the paper.

\section{SySTEM MODEL}

In this section, we introduce the observation models representing the forward and TR probing steps of the estimation algorithms. A known complex bandpass signal $f(t) \exp \left(j \omega_{c} t\right)$ ( $\omega_{c}$ denoting the angular carrier frequency), transmitted by 
element $k$ of Array $A$, is backscattered by the target with unknown spatial location $\boldsymbol{\alpha}_{t}$. After down conversion, the observation recorded by the $n$ 'th element of Array $A$ is modeled as a sum of several scaled, time delayed echos of the probing signal received through multiple paths resulting from among other factors the clutter and/or the medium boundaries as

$r_{(n, k)}(t)=\sum_{i=1}^{M_{(n)}} X_{(n, k, i)} f\left(t-\tau_{(1, k, i)}-\Delta \tau_{(n, k, i)}\right)+v_{n}(t)$,

for $t \in\left\{t_{1}, \cdots, t_{N}\right\}$. Subscript $(n, k, i)$ in Eq. (1) signifies that the echo from the $i$ 'th multipath is received by recording element $n$ when element $k$ of Array $A$ probes the channel. The remaining notation in Eq. (1) is defined as follows.

$M_{(n)} \quad$ Order of multipaths associated with element $n$, $X_{(n, k, i)} \quad$ Attenuation factor for multipath $i$ connecting receiving element $n$ to transmitting element $k$,

$\tau_{(1, k, i)} \quad$ Reference delay associated with path $i$ between transmitting element $k$ and receiving element 1 ,

$\Delta \tau_{(n, k, i)} \quad$ Interelement delay in excess of $\tau_{(1, k, i)}$ between elements $n$ and $k$ for path $i$,

and $v_{n}(t) \quad$ Observation noise.

The order $M_{(n)}$ of the multipath is assumed to be the same for all array elements $\left(M_{(n)}=M\right)$ and is known a priori. We also assume a linear array such that attenuation $X_{(n, k, i)}=X_{(i)}$, i.e., the attenuation factors are the same for all pairs $(n, k)$ of array elements for a given multipath $i$, and the interelement delay $\Delta \tau_{(n, k, i)}=|n-k| \times d \sin \theta / c$ for path $i$ is measured with respect to the first element $(n=1)$ of the array. Symbol $d$ denotes the interelement spacing for linear array $A$ and $c$ is the propagation speed. In the frequency domain, Eq. (1) is given by

$$
\begin{aligned}
r_{(n, k)}(\omega) & =\sum_{i=1}^{M} e^{-j \omega \Delta \tau_{(n, k, i)}} X_{(n, k, i)} e^{-j \omega \tau_{(1, k, i)}} F(\omega) \\
& +v_{n}(\omega) .
\end{aligned}
$$

Using the vector-matrix format, Eq. (2) is represented as

$$
\boldsymbol{r}_{k}(\omega)=\boldsymbol{A}(\boldsymbol{\Theta}) \boldsymbol{X} \boldsymbol{\Gamma}_{k}(\omega) F(\omega)+\boldsymbol{v}(\omega),
$$

where $\boldsymbol{A}(\Theta)$ defines the $(P \times M)$ DOA matrix containing all possible $M$-steering vectors, i.e.,

$\boldsymbol{A}(\boldsymbol{\Theta})=\left[\begin{array}{ccc}1 & \cdots & 1 \\ e^{-j \omega d \sin \theta_{1} / c} & \cdots & e^{-j \omega d \sin \theta_{M} / c} \\ \vdots & \ddots & \vdots \\ \underbrace{e^{-j \omega(P-1) d \sin \theta_{1} / c}}_{a\left(\boldsymbol{\theta}_{1}\right)} & \cdots & \underbrace{e^{-j \omega(P-1) d \sin \theta_{M} / c}}_{\boldsymbol{a}\left(\boldsymbol{\theta}_{M}\right)}\end{array}\right]$,

$\boldsymbol{X}$ represents the $(M \times M)$ attenuation matrix given by

$$
\boldsymbol{X}=\left[\begin{array}{llll}
X_{(1)} & & & \\
& X_{(2)} & & \\
& & \ddots & \\
& & & X_{(M)}
\end{array}\right],
$$

and notation $\boldsymbol{\Gamma}_{k}(\omega)$ is the $(M \times 1)$ multipath delay vector

$$
\boldsymbol{\Gamma}_{k}(\omega)=\left[e^{-j \omega \tau_{(1, k, 1)}}, e^{-j \omega \tau_{(1, k, 2)}}, \cdots, e^{-j \omega \tau_{(1, k, M)}}\right]^{T} .
$$

The covariance matrix of the noise $\boldsymbol{v}(\omega)$ in Eq. (3) is given by $\sigma_{v}^{2} \mathbf{I}_{P}$. Symbols $F(\omega), \boldsymbol{v}(\omega)$, and $\boldsymbol{r}_{k}(\omega)$ are, respectively, the discrete Fourier transforms of the probing signal $f(t)$, observation noise vector $\left\{v_{n}(t)\right\}$ and observation vector $\left\{r_{(n, k)}(t)\right\}$ obtained by ordering the noise and observation entries $(1 \leq n \leq P)$. Note that the channel response matrix $\mathbf{H}(\omega)$ defined as

$$
\forall k, n=1, \cdots, P, \quad \mathbf{H}(\omega) \triangleq\left\{H_{n k}\left(\omega ; A_{n} \leftarrow A_{k}\right)\right\} .
$$

can be expressed in terms of our earlier notation as

$$
H_{n k}(\omega)=\sum_{i=1}^{M} e^{-j \omega \Delta \tau_{(n, k, i)}} X_{(n, k, i)} e^{-j \omega \tau_{(1, k, i)}} .
$$

Next, we derive the observation model for the TR stage used in the TR/DOA estimator.

\section{A. TR Observations}

Following the principle of TR, the recorded signal $\boldsymbol{r}_{k}(\omega)$ is energy normalized by a factor $g$, time reversed (equivalent to phase conjugation in the frequency domain), and retransmitted back into the medium. Denoting the TR probing signal vector by $\mathbf{z}_{k}(\omega)=g \mathbf{r}_{k}^{*}(\omega)$, the TR observation vector $\boldsymbol{y}_{k}(\omega)$ is given by

$$
\boldsymbol{y}_{k}(\omega)=\sum_{j=1}^{P} \boldsymbol{A}(\boldsymbol{\Theta}) \boldsymbol{X} \boldsymbol{\Gamma}_{j}(\omega) z_{(k, j)}(\omega)+\boldsymbol{\zeta}(\omega),
$$

where $z_{(k, j)}(\omega)$ refers to the $j$ 'th element of the TR probing signal $\mathbf{z}_{k}(\omega)$ and $\boldsymbol{\zeta}(\omega)$ denotes noise with a covariance of $\sigma_{\zeta}^{2} \mathbf{I}_{P}$. In the vector-matrix format, the TR observation is expressed as Eq. (10), where $\mathbf{1}_{M}$ refers to a unit vector of dimension $M$ with all entries equal to 1 . The block matrix containing $\boldsymbol{A}(\Theta)$ is of order $(P \times P M)$, while the block matrix containing $\boldsymbol{X}$ is square with dimensions $(P M \times P M)$. The operation $\operatorname{diag}\left(\boldsymbol{\Gamma}_{n}(\omega)\right),(1 \leq n \leq P)$, represents a square matrix with elements of $\boldsymbol{\Gamma}_{n}(\omega)$ on the main diagonal of the resulting matrix. Based on these observation models, the conventional and TR/DOA estimators are explained next.

\section{DOA ESTIMATORS}

This section presents the wideband Capon estimation algorithm, which is applied to an active array (passive target) setup for DOA estimation in a rich multipath environment.

\section{A. Conventional Wideband Capon}

Following Eq. (3), we divide the frequency spectrum of the forward observations $\boldsymbol{r}_{k}(\omega)$ into $Q$ frequency bins. Denoting the frequency component of the observation in the $q$ 'th bin as $\boldsymbol{r}_{k}\left(\omega_{q}\right)$, the corresponding $q^{\prime}$ th bin spatial covariance matrix is given by

$$
\begin{aligned}
\mathbf{R}_{\boldsymbol{r}_{k}}\left(\omega_{q}\right) & =\mathbb{E}\left\{\boldsymbol{r}_{k}\left(\omega_{q}\right) \boldsymbol{r}_{k}^{H}\left(\omega_{q}\right)\right\} \\
& \approx \frac{1}{N_{q}} \sum_{m=1}^{N_{q}} \boldsymbol{r}_{k}\left(\omega_{q, m}\right) \boldsymbol{r}_{k}^{H}\left(\omega_{q, m}\right)
\end{aligned}
$$




$$
\underbrace{\left[\begin{array}{c}
y_{(k, 1)}(\omega) \\
\vdots \\
y_{(k, P)}(\omega)
\end{array}\right]}_{\boldsymbol{y}_{k}(\omega)}=[\boldsymbol{A}(\boldsymbol{\Theta}), \boldsymbol{A}(\boldsymbol{\Theta}), \cdots, \boldsymbol{A}(\boldsymbol{\Theta})] \times
$$

$$
\left[\begin{array}{ccc}
\boldsymbol{X} & & \\
& \ddots & \\
& & \boldsymbol{X}
\end{array}\right]\left[\begin{array}{lll}
\operatorname{diag}\left(\boldsymbol{\Gamma}_{1}(\omega)\right) & & \\
& \ddots & \\
& & \operatorname{diag}\left(\boldsymbol{\Gamma}_{P}(\omega)\right)
\end{array}\right]\left[\begin{array}{c}
z_{(k, 1)}(\omega) \mathbf{1}_{M} \\
\vdots \\
z_{(k, P)}(\omega) \mathbf{1}_{M}
\end{array}\right]+\boldsymbol{\zeta}(\omega)
$$

which, as shown by the lower expression, is estimated in practice from the $N_{q}$ frequency samples of the observation vector $\boldsymbol{r}_{k}\left(\omega_{q}\right)$ within bin $q$. The Capon algorithm attempts to minimize the power contributed by noise and other interference signals coming from directions other than the desired direction while maintaining a fixed gain in the direction under consideration. In our case, this direction is the the direct path DOA $\left(\theta_{1}\right)$. Expressing Capon as a minimization problem

$$
\min _{\mathbf{w}} \mathbf{w}^{H}\left(\omega_{q}, \theta\right) \mathbf{R}_{\boldsymbol{r}_{k}}\left(\omega_{q}\right) \mathbf{w}\left(\omega_{q}, \theta\right)
$$

subject to the constraint $\mathbf{w}^{H}\left(\omega_{q}, \theta\right) \boldsymbol{a}\left(\omega_{q}, \theta\right)=1$, with the steering vector $\boldsymbol{a}\left(\omega_{q}, \theta\right)$ defined previously in Eq. (4), results in the following solution for the weight vector

$$
\mathbf{w}\left(\omega_{q}, \theta\right)=\frac{\mathbf{R}_{\boldsymbol{r}_{k}}^{-1}\left(\omega_{q}\right) \boldsymbol{a}\left(\omega_{q}, \theta\right)}{\boldsymbol{a}^{H}\left(\omega_{q}, \theta\right) \mathbf{R}_{\boldsymbol{r}_{k}}^{-1}\left(\omega_{q}\right) \boldsymbol{a}\left(\omega_{q}, \theta\right)}
$$

where $\theta$ is the search angle. The narrowband power spectrum is then given by

$$
\mathbb{P}\left(\omega_{q}, \theta\right)=\frac{1}{\boldsymbol{a}^{H}\left(\omega_{q}, \theta\right) \mathbf{R}_{\boldsymbol{r}_{k}}^{-1}\left(\omega_{q}\right) \boldsymbol{a}\left(\omega_{q}, \theta\right)} .
$$

Combining results from different bins, the geometrically averaged wideband capon has the power spectrum

$$
\mathbb{Q}(\theta)=\prod_{q=1}^{Q} \frac{1}{\boldsymbol{a}^{H}\left(\omega_{q}, \theta\right) \mathbf{R}_{\boldsymbol{r}_{k}}^{-1}\left(\omega_{q}\right) \boldsymbol{a}\left(\omega_{q}, \theta\right)} .
$$

The peaks in spectrum $\mathbb{Q}(\theta)$ correspond to the estimated values of the DOA's.

\section{B. TR Wideband Capon}

Following Eq. (9), we apply the same procedure as in the conventional Capon to the TR observations $\boldsymbol{y}_{k}(\omega)$. Denoting the TR spatial covariance matrix in the q'th frequency bin as $\mathbf{R}_{\boldsymbol{y}_{k}}=\mathbb{E}\left\{\boldsymbol{y}_{k}\left(\omega_{q}\right) \boldsymbol{y}_{k}^{H}\left(\omega_{q}\right)\right\}$, the TR Capon takes the form

$$
\begin{array}{rr}
\min _{\mathbf{w}_{T R}} & \mathbf{w}_{T R}^{H}\left(\omega_{q}, \theta\right) \mathbf{R}_{\boldsymbol{y}_{k}}\left(\omega_{q}\right) \mathbf{w}_{T R}\left(\omega_{q}, \theta\right) \\
\text { subject to } & \mathbf{w}_{T R}^{H}\left(\omega_{q}, \theta\right) \boldsymbol{a}_{T R}\left(\omega_{q}, \theta\right)=1,
\end{array}
$$

in which $\boldsymbol{a}_{T R}(\theta)$ is the steering vector during the TR step and is calculated from Eq. (9) in terms of $\boldsymbol{a}(\theta)$. Due to lack of space, we include the derivation for $\boldsymbol{a}_{T R}(\theta)$ for a 2-path model used in our simulations. The derivation is generalizable to any $M$-order multipath. Based on the following 2-path model

$$
\begin{aligned}
\boldsymbol{r}_{k}(\omega) & =\left[\boldsymbol{a}\left(\theta_{1}\right) X_{(1)} e^{-j \omega \tau_{(1, k, 1)}}\right. \\
& \left.+\boldsymbol{a}\left(\theta_{2}\right) X_{(2)} e^{-j \omega \tau_{(1, k, 2)}}\right] F(\omega)+\boldsymbol{v}(\omega)
\end{aligned}
$$

the TR observations (Eq. (9)) are given by

$$
\begin{aligned}
& \boldsymbol{y}_{k}(\omega)=g \boldsymbol{a}\left(\theta_{1}\right) X_{(1)} e^{-j \omega \tau_{(1, k, 1)}} F^{*}(\omega) \times \\
& {\left[\sum_{i=1}^{P} a_{i}^{*}\left(\theta_{1}\right) X_{(1)}^{*} e^{+j \omega \tau_{(1, k, 1)}}+a_{i}^{*}\left(\theta_{2}\right) X_{(2)}^{*} e^{+j \omega \tau_{(1, k, 2)}}\right]} \\
& +g \boldsymbol{a}\left(\theta_{2}\right) X_{(2)} e^{-j \omega \tau_{(1, k, 2)} F^{*}(\omega) \times} \\
& \underbrace{\left[\sum_{i=1}^{P} a_{i}^{*}\left(\theta_{1}\right) X_{(1)}^{*} e^{+j \omega \tau_{(1, k, 1)}}+a_{i}^{*}\left(\theta_{2}\right) X_{(2)}^{*} e^{+j \omega \tau_{(1, k, 2)}}\right]}_{\boldsymbol{B}(\omega, \theta)} \\
& \quad+\boldsymbol{\zeta}(\omega) .
\end{aligned}
$$

For a 2-path model, the steering vector $\boldsymbol{a}_{T R}(\theta)$ is given by

$$
\boldsymbol{a}_{T R}(\omega, \theta)=\boldsymbol{a}(\omega, \theta) \times \boldsymbol{B}(\omega, \theta) .
$$

In other words, the TR operation provides a mechanism for beamspace beamforming, which focuses the beam towards the DOA $\theta_{1}$ using multipath to its advantage. Therefore, we expect more accurate DOA estimation using this approach. The DOA estimates are obtained from the peaks of the TR spectrum

$$
\mathbb{Q}_{T R}(\theta)=\prod_{q=1}^{Q} \frac{1}{\boldsymbol{a}_{T R}^{H}\left(\omega_{q}, \theta\right) \mathbf{R}_{\boldsymbol{y}_{k}}^{-1}\left(\omega_{q}\right) \boldsymbol{a}_{T R}\left(\omega_{q}, \theta\right)} .
$$

\section{CRBS FOR THE DOA ESTIMATORS}

In this section, we present the CRBs for both the conventional and TR DOA and range estimators for a passive stationary target. Parameter $\boldsymbol{\alpha}_{t}=\left[\begin{array}{ll}R_{t}, & \theta_{t}\end{array}\right]^{T}$ is a vector containing the range $\left(R_{t}\right)$ and DOA $\left(\theta_{t}\right)$ of the target. In range estimation, the range of the target is typically measured in terms of the round-trip time $\tau$ for the probing waveform to travel out to the target from the transmitter and back to the receiver. Theorem 1 derives a lower bound on the accuracy of the conventional DOA and range estimators based on Eq. (3). Likewise, Theorem 2 expresses the lower bound on the accuracy of the TR DOA and range estimators based on Eq. (9). The proofs of the two theorems are included in [8] 
presented earlier this year. While [8] is limited to the CRBs, this paper designs the Capon based TR/DOA estimator and plots the actual MSE of the estimator in addition to the CRBs.

Theorem 1. The CRB for the direction of arrival and the range of the targets based on the forward observation vector $\boldsymbol{r}_{k}(\omega)(E q .(3))$ for the conventional estimator is given by

$$
C R B_{C V}(\boldsymbol{\alpha})^{-1}=\frac{N}{\pi \sigma_{v}^{2}} \Re\left\{\int|F(\omega)|^{2} \mathbf{D}^{H} \mathbf{D} d \omega\right\},
$$

where the $(P \times 2)$ derivative matrix $\mathbf{D}$ is

$$
\mathbf{D}=\left[\frac{\partial \mathbf{h}_{k}}{\partial R_{t}} \frac{\partial \mathbf{h}_{k}}{\partial \theta_{t}}\right]
$$

and the vector $\mathbf{h}_{k}=\mathbf{H}(\omega) \mathbf{e}_{k}$ is the channel response vector in the forward probing stage. The matrix $\mathbf{D}$ groups all derivatives of the multipath response vector $\mathbf{h}_{k}$ with respect to the source location parameters $R_{t}$ and $\theta_{t}$. Based on Eq. (3) the partial derivatives of the multipath channel response in Eq. (21) can be further simplified to

$$
\begin{aligned}
\frac{\partial \mathbf{h}_{k}}{\partial R_{t}} & =\boldsymbol{A}(\boldsymbol{\Theta}) \boldsymbol{X}\left(\frac{\partial \boldsymbol{\Gamma}_{k}}{\partial R_{t}}\right) \\
\frac{\partial \mathbf{h}_{k}}{\partial \theta_{t}} & =\left(\frac{\partial \boldsymbol{A}(\boldsymbol{\Theta})}{\partial \theta_{t}}\right) \boldsymbol{X} \boldsymbol{\Gamma}_{k}
\end{aligned}
$$

The CRBs for the TR DOA and range estimators are considered next.

Theorem 2. The CRB of the DOA and range of the targets based on the TR observation vector $\mathbf{y}_{k}(\omega)$ (Eq. (9)) for the TR estimators is given by

$$
C R B_{T R}(\boldsymbol{\alpha})^{-1}=\frac{N g^{2}}{\pi \sigma_{\zeta}^{2}} \Re\left\{\int|F(\omega)|^{2} \mathbf{E}^{H} \mathbf{E} d \omega\right\},
$$

where the $(P \times 2)$ derivative matrix $\mathbf{E}$ is given by

$$
\mathbf{E}=\left[\frac{\partial \mathbf{t}_{k}}{\partial R_{t}} \frac{\partial \mathbf{t}_{k}}{\partial \theta_{t}}\right],
$$

$g$ is the TR energy normalization factor, and vector $\mathbf{t}_{k}=$ $\mathbf{T}(\omega) \mathbf{e}_{k}$ is the k'th column of the TR matrix $\mathbf{T}(\omega)=$ $\mathbf{H}(\omega) \mathbf{H}^{*}(\omega)$, which represents the channel response in the $T R$ stage. The matrix $\mathbf{E}$ groups all the derivatives of the time reversal multipath response vector $\mathbf{t}_{k}$ with respect to the source location parameters $R_{t}$ and $\theta_{t}$.

In Theorems 1 and 2 , vector $\mathbf{e}_{k}$ is a column vector with all entries except the $k$ 'th entry equal to 0 . The $k$ 'th entry is 1. These theorems provide the lower MSE bound on the error variance of the estimators and are plotted along with the actual performance characteristics of the DOA estimators in the experimental section.

\section{EXPERIMENTAL Simulations}

In this section, we investigate the performance of the TR/DOA estimator and compare it with the conventional DOA estimator. The implementations of the DOA estimators are based on the Capon algorithm as described previously in Section III. Our comparisons are based on the following

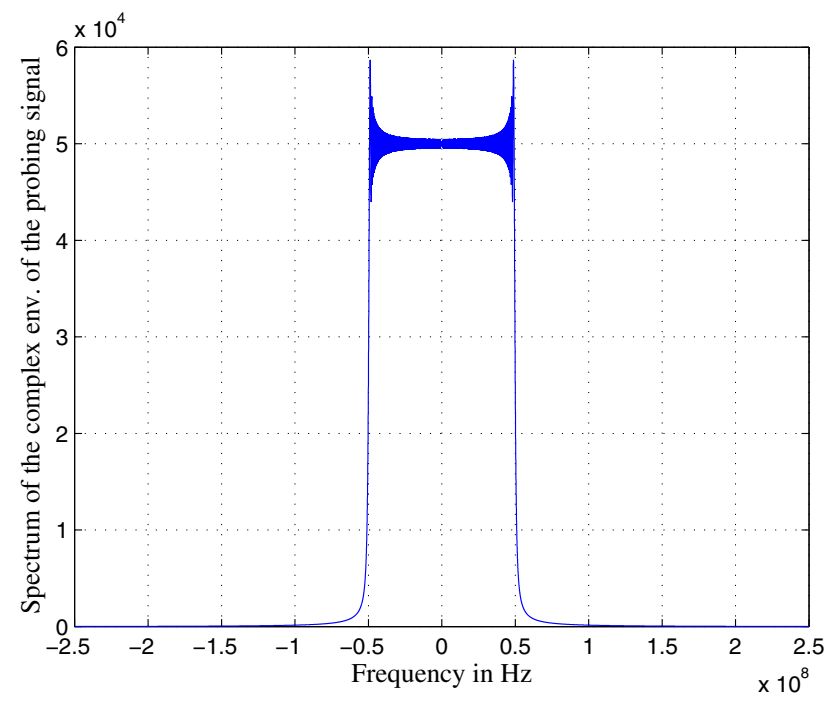

Fig. 1. Spectrum of the probing signal

four characteristics: (i) Mean square errors (MSE) of the estimators based on Monte Carlo simulations; (ii) Resolution capability; (iii) Ability to accurately estimate the DOA of a target in the presence of multipath, and; (iv) the CRBs illustrating the potential performance possible with the two approaches. For simplicity of implementation, we assume a uniform linear array compromising of $P=10$ sensors with interelement sensor spacing $d$ set to $\lambda_{\min } / 2$, where $\lambda_{\min }$ is the minimum wavelength present in the broadband signal. The probing signal is assumed to be a pulse with linear frequency modulation (LFM), $\left(f(t)=\tilde{f}(t) e^{j \omega_{0} t}\right)$, where the angular frequency $\omega_{0}=2 \pi f_{0}$ and the base chirp frequency $f_{0}$ equals $5 \mathrm{GHz}$. The complex envelope $\tilde{f}(t)$ of the probing signal is given by

$$
\tilde{f}(t)=\frac{1}{\sqrt{\tau}_{0}} \operatorname{Rect}\left(\frac{t}{\tau_{0}}\right) e^{j \pi \mu t^{2}} .
$$

The spectrum of the probing signal is plotted in Fig. 1. For the conventional Capon, Eq. (1) is used to model the 2-path received signals $r_{(n, k)}(t)$ with DOA's set to $\left\{40^{\circ},-30^{\circ}\right\}$ and the corresponding attenuation factors given by $\{1,0.4\}$. The values of the reference delays for the two components are $\{7,7.33\} \mathrm{ms}$. Observation noise with different variances is added to simulate a variety of signal-to-noise ratios (SNR). The conventional DOA estimator estimates the DOA based on the observation $r_{(n, k)}(t)$. The TR observation is modeled on Eq. (9) with same values for the DOAs and attenuation factors as were used in the conventional Capon. The power spectra produced by the conventional and TR/DOA estimators are plotted in Fig. 2, where for reference we plot two vertical lines at $\left\{40^{\circ}\right.$ and $\left.-30^{\circ}\right\}$ corresponding to the actual values of the DOAs corresponding to ground reality. We note that the TR/DOA estimator produces a better result on all four performance characteristics mentioned before. There are only two peaks present in the TR/DOA spectrum (shown by the dotted line), which corresponds to the order of the 


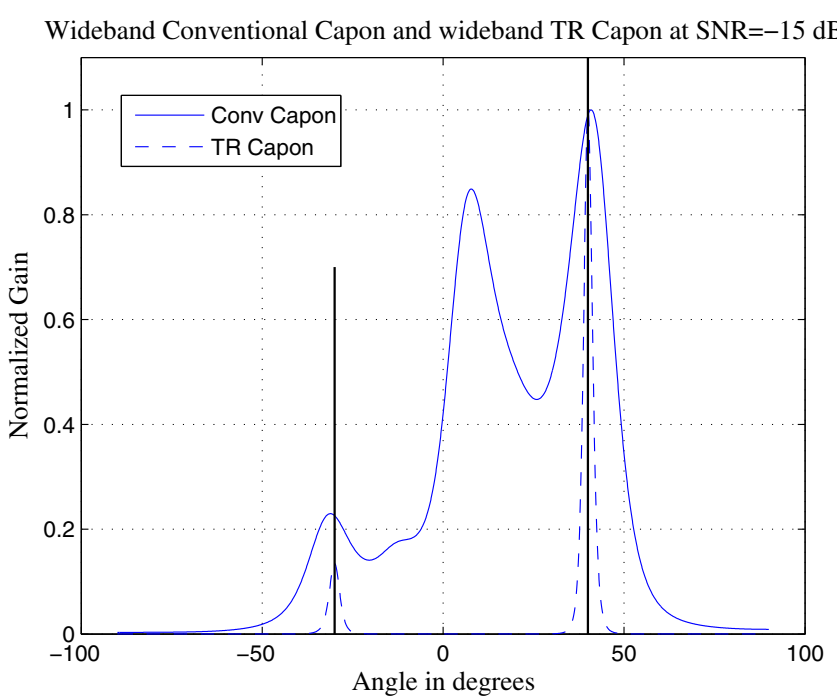

Fig. 2. Output of the Capon Estimator based on: (a) Conventional Measurement, and; (b) TR Measurement, for a 2-path propagation model with respective DOAs set to $\left\{40^{\circ},-30^{\circ}\right\}$.

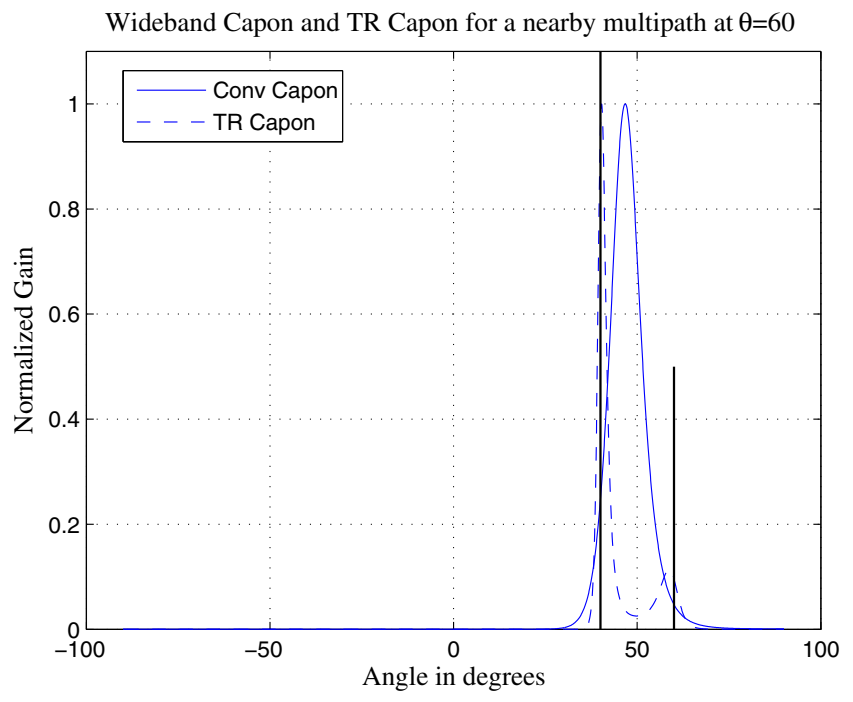

Fig. 3. Same as Fig. 2 except that the DOAs are set to $\left\{40^{\circ}, 60^{\circ}\right\}$.

multipath in the received signal. The power spectrum for the conventional capon shows an erroneous third peak around $6^{\circ}$. In addition, the TR/DOA estimator is more accurate with the peaks in its spectrum observed closer to the simulated DOAs with much finer resolutions. A second comparison is included in Fig. 3 for DOAs set to $\left\{40^{\circ}, 60^{\circ}\right\}$. While the TR/DOA estimator is able to isolate the two DOAs, the spectrum of the conventional DOA estimator shows only one peak illustrating the superior performance of the TR/DOA estimator. In Fig. 4, the performance curves for the two estimators along with their CRBs are plotted. Comparing the CRBs, we note that the TR/DOA estimator has a lower bound and, therefore, has a better potential of providing superior performance than the conventional DOA estimator. Comparing the actual performance curves, we observe that the MSE for

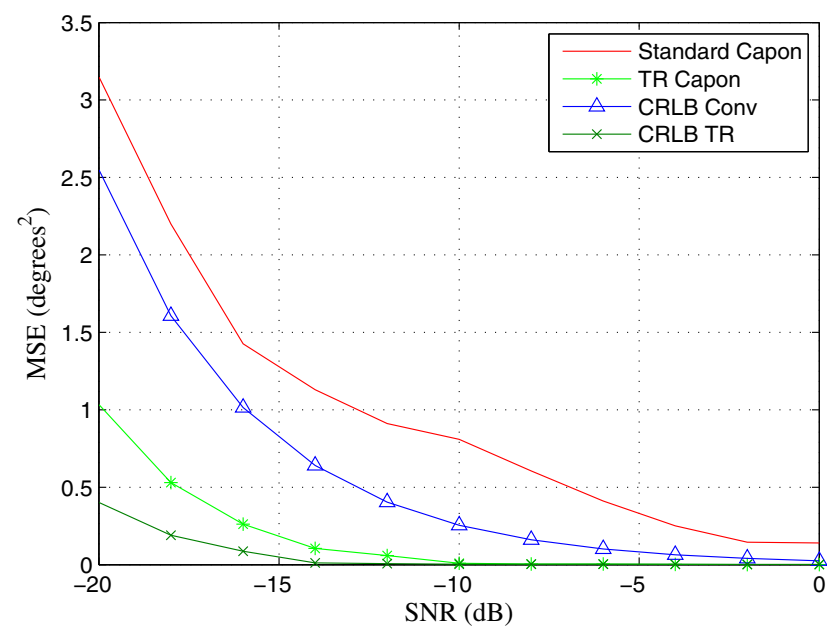

Fig. 4. Performance curves for the conventional and TR/DOA estimators. For comparison, the plots for the CRB's are also included in the figure.

the TR/DOA estimator is much lower in comparison with the conventional DOA estimator especially for low SNR's below $-5 \mathrm{~dB}$.

\section{SUMmARY AND FUtURE WORK}

In this paper, we applied TR to DOA estimation. The effect of coupling TR to DOA estimation is studied through theoretical Cramér-Rao bound (CRB) analysis as well as numerical simulations. In our simulations, we observe that the proposed TR/DOA estimator is more accurate in its estimation, provides higher resolution, and exhibits a lower MSE for a variety of SNRs that we tested. Compared to its conventional counterpart, our analytical comparison based on the CRBs supports our experimental results and illustrates the potential of significantly better performance with the proposed TR/DOA approach.

\section{REFERENCES}

[1] L. C. Godara, "Applications of Antenna Arrays to Mobile Communications, Part I: Performance Improvement, Feasibility, and System Considerations," Proceedings of the IEEE, vol. 85, no. 7, pp. 1031-1060, Jul. 1997.

[2] V. Tsoulos, "Smart antennas for mobile communication systems," Electronics and Communication Engineering Journal, vol. 11, No. 2, pp. 8494, Apr. 1999.

[3] M. Fink, "Time Reversal Acoustics," Physics Today, vol. 39, no. 5, 1997, pp. $555-566$.

[4] J. M. F. Moura and Y. Jin, "Detection by time reversal: single antenna,"IEEE Trans. on Signal Processing, vol. 55, 2007, pp. 187-201.

[5] N. J. Roseveare and M. R. Azimi-Sadjadi, "Capon beamspace beamforming for distributed acoustic arrays," in Proceedings of SPIE, vol. 6562, 2007.

[6] J. Capon, "High-resolution frequency-wavenumber spectrum analysis," Proceedings of the IEEE, vol. 57, no. 8, pp. 1408-1418, Aug. 1969.

[7] J. Li, P. Stoica, and Z. Wang, "On robust Capon beamforming and diagonal loading," IEEE Transactions on Signal Processing, vol. 51, no. 7, pp. 1702-1715, July 2003.

[8] F. Foroozan and A. Asif, "Cramér-Rao Bound for Time Reversal Active Array Direction of Arrival Estimators in Multipath Environments," in Proceedings of the IEEE International Conference on Acoustics, Speech, and Signal Processing (ICASSP), pp. 2646-2649, 2010, Dallas. 